\section{Incapacidade laboral entre trabalhadores do ramo Correios: incidência, duração e despesa previdenciária em 2008}

\author{
Work-related disability among postal employees: \\ incidence, duration, and social security costs \\ in 2008
}

\author{
Incapacidad laboral entre los trabajadores de \\ Correos: incidencia, duración y gastos en \\ pensiones durante 2008
}

\author{
1 Universidade de Brasília, \\ Brasília, Brasil. \\ Correspondência \\ F. A. N. Mascarenhas \\ Universidade de Brasília. \\ SQS 404, bloco P, apto. 308 \\ Brasília, DF \\ 70238-190, Brasil. \\ flavia_neves@hotmail.com
}

\begin{abstract}
This study analyzed the characteristics of Brazilian postal workers that received sick leave benefits in 2008. The databases were from the Unified Benefits System (SUB) and the National Registry of Social Information (CNIS). The incidence rate was 556.5 benefits per 10,000 employees, and the leading causes of work-related sick leave were injuries, musculoskeletal disorders, and mental disorders. Areas most frequently reported in injuries were knees and legs, wrists and hands, ankles and feet, and shoulders and arms, with higher incidence rates in men. Women were more affected by musculoskeletal disorders and mental disorders. Average sick leave lasted longer in men, and the incidence of benefits increased with age. The States with the highest incidence rates were Mato Grosso do Sul, Goiás, and Santa Catarina, and security benefits averaged BRL 1,847.00. Postal work may involve additional risk of injuries to the limbs, due to the long distances carrying heavy weight, assault, and dog bites.
\end{abstract}

Postal Service; Absenteeism; Occupational Diseases; Occupational Health
Flávia Alves Neves Mascarenhas 1

Anadergh Barbosa-Branco ${ }^{1}$

\section{Resumo}

Analisou-se o perfil dos trabalhadores do ramo de atividade Correios que receberam benefícios auxílio-doença, no Brasil, em 2008. Os dados originaram-se do Sistema Único de Benefícios (SUB) e do Cadastro Nacional de Informações Sociais (CNIS). A incidência foi de 556,5 benefícios por 10 mil empregados, e as principais causas de afastamento foram traumatismos, doenças osteomusculares e transtornos mentais. Locais de traumas mais notificados foram joelho e perna, punho e mão, tornozelo e pé, ombro e braço, com as maiores incidências em homens. Mulheres foram as mais acometidas por doenças osteomusculares e transtornos mentais. A duração do auxílio-doença foi maior entre homens, havendo aumento da incidência de benefícios com o envelhecimento. Os estados de Mato Grosso do Sul, Goiás, Santa Catarina foram os de maiores incidências, e a despesa previdenciária foi de R\$1.847,00. É possível que a atividade postal apresente riscos adicionais de acidentes em extremidades devido ao excesso de peso carregado por longas distâncias, risco de assaltos ou de acidente por mordedura de cães.

Serviços Postais; Absenteísmo; Doenças Profissionais; Saúde do Trabalhador 


\section{Introdução}

O absenteísmo-doença tem um impacto importante no campo social, econômico, ocupacional e da saúde 1,2,3, particularmente quando gera incapacidade para o trabalho, temporária ou indefinida.

Do ponto de vista econômico, as repercussões decorrentes da incapacidade laboral atingem diversos personagens 1 : o trabalhador, com a diminuição dos vencimentos; o empregador, responsável pelo pagamento dos primeiros 15 dias de afastamento do trabalho, arcando ainda com a substituição do trabalhador incapacitado; e também a Previdência Social, com o pagamento dos benefícios a partir do 16o dia.

A Organização Internacional do Trabalho (OIT) estima que, em 2013, os acidentes e doenças relacionados com o trabalho resultaram em uma perda anual de $4 \%$ do produto interno bruto (PIB) mundial. O valor, equivalente a cerca de 2,8 trilhões de Dólares, deveu-se a custos diretos (relacionados com o pagamento dos salários) e a custos indiretos ou ocultos, de difícil quantificação e, com frequência, subestimados 4,5.

Em 2009, o custo das doenças relacionadas ao trabalho na União Europeia foi estimado em, pelo menos, 145 bilhões de Euros por ano ${ }^{6}$. Na Coreia do Sul, em 2011, o custo das doenças musculoesqueléticas foi de 6,89 bilhões de Dólares, o que representou $0,7 \%$ do PIB desse país 4 . Na Nova Zelândia, essas patologias geraram um custo estimado de mais de 4,71 bilhões de Dólares em 2009, constituindo cerca de um quarto do custo total anual dos cuidados de saúde 4 .

No Brasil, não há estimativas específicas do custo direto relacionado ao absenteísmo-doença para a economia. Além disso, a incapacidade para o trabalho no Brasil é ainda caracterizada pela subnotificação tanto dos agravos à saúde quanto de sua relação com o trabalho 7 .

Para que ocorra a concessão do auxílio-doença, não basta apenas a presença do agravo, mas esse agravo deve gerar incapacidade para o trabalho ${ }^{8}$, conceito que varia significativamente, dependendo dos parâmetros legais estabelecidos por cada sistema previdenciário 2. No Brasil, no contexto da Previdência Social, a incapacidade para o trabalho é estabelecida formalmente a partir de licença médica com duração superior a 15 dias consecutivos, da solicitação de benefício auxílio-doença junto ao Instituto Nacional de Seguro Social (INSS) e da caracterização da incapacidade pela perícia médica institucional.

Vários fatores de risco têm sido associados ao absenteísmo-doença e à incapacidade para o trabalho, com destaque para sexo, idade e ramo de atividade 2 . Do ponto de vista epidemiológico, tra- balhadores do sexo feminino e mais velhos apresentam maior incidência de auxílio-doença e também duração mais prolongada do benefício 2,9.

Uma revisão sobre os fatores-chave do trabalho associados a problemas de saúde mental e absenteísmo-doença apontou, como importantes fatores nesse processo 10 , as longas jornadas, a sobrecarga e a pressão laborais; a falta de controle sobre o trabalho; a falta de participação na tomada de decisão; o baixo suporte social; a gestão insegura e a insegurança empregatícia.

No Brasil, as principais causas clínicas de incapacidade para o trabalho são lesões, envenenamento e algumas outras consequências de causas externas; doenças do sistema osteomuscular e do tecido conjuntivo, além dos transtornos mentais e comportamentais 2 .

Quanto às categorias diagnósticas específicas, foram encontradas grandes diferenças nas taxas de prevalência de incapacidade para o trabalho entre as diversas atividades econômicas, tanto para agravos relacionados ao trabalho, como para os não relacionados 7,11,12,13. Publicação recente demostrou a importância da atividade econômica como um indicador de risco ambiental para a incapacidade para o trabalho ${ }^{2}$.

Esse mesmo estudo ${ }^{2}$, ao analisar os ramos de atividades com as maiores incidências de incapacidade para o trabalho, de acordo com os principais grupos de causas clínicas, demonstrou que o ramo de atividade Correios figurou entre as vinte maiores incidências no Brasil; em relação às doenças mentais e comportamentais, esse ranking sobe para o quarto lugar. Tais resultados apontam para a importância de aprofundar o conhecimento sobre as causas de incapacidade nesse ramo, bem como de identificar os fatores associados a ela, o que pode fornecer importantes dados para os gestores públicos, que precisam lidar com suas consequências. Pode, também, subsidiar a elaboração de intervenções que efetivamente reduzam os riscos no local de trabalho 14 .

A OIT, em 2003, numa publicação específica sobre o serviço postal, alertou que os carteiros figuram entre as atividades consideradas de "alto risco" de exposição à violência e estresse 15 . Segundo o Sindicato dos Trabalhadores da Comunicação do Reino Unido, as principais causas de estresse entre os trabalhadores dos Correios, em 2001, foram má gestão, excesso de carga de trabalho, falta de pessoal, insegurança, monotonia, longa duração das jornadas, bullying e falta de controle sobre o trabalho executado 15 .

O desenvolvimento tecnológico e as novas necessidades dos clientes exigiram grandes mudanças não só relacionadas aos produtos e serviços oferecidos, mas também, e fundamen- 
talmente, na forma como o setor postal funciona. Essas mudanças incessantes deram origem à insegurança no trabalho, à insatisfação e à tensão nas relações entre gestores, trabalhadores e representantes sindicais 16 .

No Brasil, a Empresa Brasileira de Correios e Telégrafos (ECT) é a principal representante desse ramo de atividade. Atua no segmento postal, sendo detentora da exclusividade sobre os serviços de recebimento, transporte e entrega de carta, cartão-postal e correspondência agrupada, além do recebimento, transmissão e entrega de telegramas, conforme previsto na Lei no 6.538/78 17 . Abrange, ainda, atividades nos setores de transporte e entrega de encomendas, periódicos e peças de marketing direto; no fornecimento de serviços de logística em remessas internacionais de objetos; atua como correspondente bancário, oferecendo serviços financeiros e de conveniência, como a obtenção do CPF, compra e recebimento de produtos, pagamento de aposentados e pensionistas da Previdência Social e serviço de filatelia 17 .

Os trabalhadores da ECT compõem uma população heterogênea, segundo os níveis socioeconômicos e de escolaridade, que exerce diferentes atividades no trabalho, estando, assim, exposta a fatores de risco distintos. No quadro da empresa, a profissão carteiro representa mais de $50 \%$ do efetivo de empregados. Em 2008, os trabalhadores do sexo masculino corresponderam a 76,5\% desse efetivo, e o nível de escolaridade mais prevalente foi o ensino médio completo, com $75,2 \%$ do efetivo da empresa 17 .

A Classificação Nacional de Atividade Econômica (CNAE) representa o ramo de atividade no qual se insere cada empresa e geralmente um grupo diversificado de empresas com atividades semelhantes. O ramo "Correios e outras atividades de entrega” (CNAE 53) é um caso incomum, no qual um CNAE é representado predominantemente por uma empresa (ECT). Esse contexto permite extrapolar a análise de pesquisas sobre essa população tanto para o CNAE como um todo, quanto para essa estatal em particular.

Este estudo visa a verificar a influência de fatores pessoais e ocupacionais na incidência de benefícios auxílio-doença, por mais de 15 dias, concedidos em 2008 para os trabalhadores licenciados no ramo de "Correios e outras atividades de entrega”.

\section{Métodos}

Trata-se de estudo transversal, descritivo, conduzido a partir de duas bases de dados, ambas vinculadas ao Ministério da Previdência Social. A primeira base é representada pelo Sistema Único de Benefícios (SUB) - casos -, composta pelos registros de todos os benefícios concedidos pela Previdência Social aos trabalhadores da iniciativa privada. A segunda é originária do Cadastro Nacional de Informações Sociais (CNIS) e representa o conjunto de trabalhadores declarados mensalmente por cada empresa (população exposta) à Previdência Social.

Nesta pesquisa, foram selecionados, em ambas as bases, os dados referentes aos trabalhadores declarados pelas empresas do ramo de atividade econômica "Correios e outras atividades de entrega”, em 2008.

No banco de dados dos benefícios concedidos aos trabalhadores do ramo em estudo, foram selecionados para análise apenas os auxílios-doença (previdenciários e acidentários) iniciados em 2008. A caracterização do ramo de atividade foi estabelecida segundo a classe patronal da atividade econômica, definida pela CNAE 2.0 (Instituto Brasileiro de Geografia e Estatística. Classificação Nacional de Atividades Econômicas, versão 2.0 - CNAE 2.0. http:/ / www. ibge.gov.br/home/estatistica/economia/classi ficacoes/cnae2.0/default.shtm, acessado em 02/ Dez/2013), sob a responsabilidade da Comissão Nacional de Classificação (CONCLA).

A população-alvo do estudo foi definida com base na média mensal dos registros de emprego declarados, em 2008, ao CNIS. Por força de lei, as empresas fornecem essas informações mensalmente, por meio eletrônico, conforme as Guias de Recolhimento do Fundo de Garantia por Tempo de Serviço (FGTS) e Informação à Previdência Social (GFIP).

No Brasil, quando um empregado segurado da Previdência Social é acometido por algum problema de saúde que o incapacite para as atividades laborais por mais de 15 dias, ele faz jus ao recebimento do auxílio-doença. O benefício possui duas naturezas: auxílio-doença previdenciário (não ocupacional e ocupacional) e auxíliodoença acidentário ou por acidente de trabalho, relacionado ao meio ambiente do trabalho. Cada ocorrência (previdenciária ou acidentária) que implique a concessão de um benefício é registrada no SUB e recebe um número único de identificação (número do benefício). Esse número permite acessar um conjunto de informações, tais como a CNAE da empresa, além de alguns dados do empregado e do benefício, neste incluídos a espécie do benefício e o diagnóstico clínico, codificado pela 10a revisão da Classificação Internacional de Doenças (CID-10) 8,18,19.

Foram estudados todos os auxílios-doença iniciados entre 1o de janeiro e 31 de dezembro de 2008. A recorrência de incapacidade pelo 
mesmo diagnóstico em até 60 dias após o retorno do funcionário ao trabalho é vinculada ao benefício anterior, caracterizando um único benefício.

A mensuração do nexo técnico entre o trabalho e a incapacidade laboral foi feita com base nas medidas de incidência, analisando-se as razões de incidência entre as espécies de benefícios e sexos. A incidência (x 10 mil vínculos empregatícios) foi estimada pela soma dos auxíliosdoença iniciados em 2008 dividida pela média mensal dos vínculos empregatícios declarados no mesmo ano.

A duração dos benefícios (em dias) e a despesa previdenciária (em Reais) foram apresentadas por meio de interquartis (mediana e quartis $1 \mathrm{e}$ 3) e estratificadas por espécie de benefício, sexo, idade e Unidade Federativa (UF) de localização da empresa. A despesa previdenciária representa o valor total pago pelo INSS ao beneficiário, e ela foi calculada dividindo-se o salário de benefício do trabalhador por trinta e multiplicando-o pela duração do benefício em dias.

As identidades dos trabalhadores e empresas não constavam das bases de dados pesquisadas, e tratou-se de dados administrativos, analisados retrospectivamente. A pesquisa foi realizada com um termo de concordância do Ministério da Previdência Social, autorizando a coleta dos dados e sua utilização.

\section{Resultados}

Em 2008, foram concedidos 8.608 benefícios auxílio-doença à população de trabalhadores empregados no ramo de atividade econômica "Correios e outras atividades de entrega". O total de trabalhadores desse ramo cadastrados pelo CNIS foi de 154.677 pessoas, resultando em uma incidência de 556,5 auxílios-doença por $10 \mathrm{mil}$ empregados.

Quanto à espécie de benefício, predominou o auxílio-doença previdenciário (B31). Comparando entre os sexos, as mulheres apresentaram maiores incidências de benefícios previdenciários (412,1 versus 389 , 8 benefícios), contrapondo-se aos homens, com maiores incidências de acidentários (B91) (181,8 versus 144,5 benefícios).

A distribuição das incidências de benefícios auxílio-doença segundo os capítulos da CID-10 mostra que, entre os empregados do ramo Correios, os principais grupos diagnósticos foram lesões, envenenamento e algumas outras consequências de causas externas (capítulo XIX); doenças do sistema osteomuscular e do tecido conjuntivo (capítulo XIII), além dos transtornos mentais e comportamentais (capítulo V) (Tabela 1).
A análise dos grupos diagnósticos (capítulos CID-10) mostrou clara diferença entre as causas que incapacitam homens e mulheres. Observouse que essas diferenças independem da espécie de benefícios. Entre as mulheres, predominou o grupo das doenças do sistema osteomuscular e do tecido conjuntivo (capítulo XIII), enquanto, entre os homens, o predomínio foi do grupo lesões, envenenamento e algumas outras consequências de causas externas (capítulo XIX). Neste grupo, a razão de masculinidade entre os sexos foi de 2,5. Essa razão mostra que a probabilidade de um trabalhador do sexo masculino apresentar incapacidade para o trabalho (auxílio-doença) decorrente desse grupo de agravos é 2,5 vezes maior do que a de uma trabalhadora.

Apesar de a razão de incidência para o conjunto dos benefícios auxílio-doença ser quase igual entre os sexos, observaram-se grandes variações entre eles quando analisados de acordo com os agrupamentos diagnósticos. Enquanto as maiores taxas de auxílios-doença entre o sexo masculino foram encontradas para todos os grupos das lesões (capítulo XIX) e das doenças do olho e anexos (capítulo VII), entre o feminino houve forte predominância dos grupos das neoplasias (capítulo II), das doenças do aparelho geniturinário (capítulo XIV) e das doenças endócrinas, nutricionais e metabólicas (capítulo IV).

A análise das razões de incidência entre as espécies de benefício também mostrou grandes diferenças entre os grupos, variando de 1,1, para as lesões, envenenamento e algumas outras consequências de causas externas, a 131,5, para as neoplasias, com uma razão de incidência geral de 2,3. Essa é taxa altamente influenciada pela elevada quantidade de lesões (capítulo XIX), que corresponde a $35,2 \%$ do total de auxílios-doença. $\mathrm{Na}$ avaliação das incidências de benefícios, segundo os agrupamentos da CID-10, sexo e espécie de benefício, nota-se a predominância das dorsopatias (M40-M54), traumatismos do joelho e da perna (S80-S89), transtornos dos tecidos moles (M60-M79) e artropatias (M00-M25) (Tabela 2). Os traumatismos são os agrupamentos mais incidentes se analisados em conjunto, e os locais de trauma mais notificados foram joelho e perna (S80-S89), punho e mão (S60-S69), tornozelo e pé (S90-S99), além de ombro e braço ( $\mathrm{S40}$ S49). As maiores incidências de traumatismos, independentemente do agrupamento, ocorrem em homens, para ambas as espécies de benefício. Por outro lado, os transtornos dos tecidos moles, os transtornos do humor (F30-F39) e os transtornos neuróticos, relacionados com o estresse e somatoformes (F40-F48), foram mais incidentes entre as mulheres. 
Incidência de benefícios auxílio-doença concedidos aos trabalhadores do ramo Correios, segundo capítulos da 10ạ revisão da Classificação Internacional de Doenças (CID-10), a espécie de beneficio e o sexo. Brasil, 2008.

\begin{tabular}{|c|c|c|c|c|c|c|c|c|c|c|c|}
\hline \multirow[t]{3}{*}{ Capítulos da CID-10 } & \multicolumn{6}{|c|}{ Espécie de benefício } & \multirow[t]{3}{*}{ Total M } & \multirow[t]{3}{*}{ Total F } & \multirow{3}{*}{$\begin{array}{c}\text { RI } \\
\text { B31/B91 }\end{array}$} & \multirow{3}{*}{$\begin{array}{c}\text { RI } \\
\text { M/F }\end{array}$} & \multirow[t]{3}{*}{ Total } \\
\hline & \multicolumn{3}{|c|}{ B31 } & \multicolumn{3}{|c|}{ B91 } & & & & & \\
\hline & M & $F$ & Total & M & $F$ & Total & & & & & \\
\hline I - Doenças infecciosas & 7,0 & 3,5 & 6,0 & 0,4 & 0,3 & 0,3 & 7,4 & 3,7 & 18,6 & 2,0 & 6,3 \\
\hline II - Neoplasias & 10,9 & 36,9 & 17,0 & 0,2 & 0,0 & 0,1 & 11,1 & 36,9 & 131,5 & 0,3 & 17,1 \\
\hline III - Doenças do sangue & 0,4 & 1,1 & 0,6 & 0,0 & 0,0 & 0,0 & 0,4 & 1,1 & - & 0,4 & 0,6 \\
\hline IV - Doenças endócrinas & 1,9 & 5,1 & 2,7 & 0,0 & 0,0 & 0,0 & 1,9 & 5,1 & - & 0,4 & 2,7 \\
\hline V-Transtornos mentais & 54,8 & 71,4 & 57,7 & 13,8 & 19,5 & 14,9 & 68,6 & 91,0 & 3,9 & 0,8 & 72,7 \\
\hline $\mathrm{VI}$ - Doenças do sistema nervoso & 6,5 & 10,2 & 7,2 & 1,3 & 3,5 & 1,8 & 7,8 & 13,6 & 4,0 & 0,6 & 9,1 \\
\hline VII - Doenças do olho e anexos & 9,1 & 3,7 & 7,6 & 0,0 & 0,0 & 0,0 & 9,1 & 3,7 & - & 2,4 & 7,6 \\
\hline VIII - Doenças do ouvido & 1,6 & 2,4 & 1,7 & 0,0 & 0,0 & 0,0 & 1,6 & 2,4 & - & 0,7 & 1,7 \\
\hline IX - Doenças do aparelho circulatório & 28,6 & 28,1 & 27,9 & 0,6 & 0,3 & 0,5 & 29,2 & 28,4 & 54,0 & 1,0 & 28,4 \\
\hline X - Doenças do aparelho respiratório & 5,9 & 4,5 & 5,4 & 0,1 & 0,5 & 0,2 & 6,0 & 5,1 & 28,0 & 1,2 & 5,6 \\
\hline XI - Doenças do aparelho digestivo & 35,3 & 25,2 & 32,1 & 0,7 & 0,0 & 0,5 & 36,0 & 25,2 & 62,1 & 1,4 & 32,6 \\
\hline XII - Doenças da pele & 4,1 & 3,5 & 3,9 & 0,3 & 0,5 & 0,3 & 4,4 & 4,0 & 12,0 & 1,1 & 4,2 \\
\hline XIII - Doenças osteomusculares & 87,7 & 101,4 & 89,3 & 50,6 & 70,4 & 54,4 & 138,3 & 171,8 & 1,6 & 0,8 & 143,7 \\
\hline XIV - Doenças do aparelho geniturinário & 9,4 & 30,5 & 14,3 & 0,3 & 0,0 & 0,2 & 9,6 & 30,5 & 73,7 & 0,3 & 14,5 \\
\hline XV - Gravidez, parto, puerpério & - & 36,1 & 36,1 & - & 0,0 & 0,0 & - & 36,1 & - & - & 36,1 \\
\hline XVII - Malformações & 0,3 & 1,1 & 0,5 & 0,0 & 0,0 & 0,0 & 0,3 & 1,1 & - & 0,2 & 0,5 \\
\hline XIX - Causas externas & 122,0 & 43,1 & 100,5 & 112,9 & 49,2 & 95,3 & 235,0 & 92,3 & 1,1 & 2,5 & 195,8 \\
\hline$X X I$ - Exames de rotina & 3,9 & 4,0 & 3,9 & 0,4 & 0,3 & 0,4 & 4,4 & 4,3 & 10,0 & 1,0 & 4,3 \\
\hline Total & 389,8 & 412,1 & 387,4 & 181,8 & 144,5 & 169,1 & 571,5 & 556,6 & 2,3 & 1,0 & 556,5 \\
\hline
\end{tabular}

B31: auxílio-doença previdenciário; B91: auxílio-doença acidentário F: feminino M: masculino; RI: razão de incidência.

Nota: os demais capítulos não foram analisados individualmente em virtude do baixo número de casos.

Segundo a Tabela 2, constata-se que todos os agrupamentos da CID-10 que tratam de traumatismos apresentaram razão de incidência entre as espécies de benefícios (B31/B91) próxima a um, apresentando importante caracterização de nexo técnico, à semelhança dos transtornos dos tecidos moles (M60-M79).

Quando se comparam as razões de incidência entre os sexos em relação aos agrupamentos diagnósticos, verifica-se que os transtornos mentais e comportamentais decorrentes do uso de substância psicoativa (F10-F19) apresentaram razão de masculinidade de 11,7 , enquanto as neoplasias benignas (D10-D36) apresentaram elevada razão de feminilidade (RP 10).

Dentre os agrupamentos da CID-10 mais incidentes, aqueles com menor caracterização de nexo técnico (relação com o trabalho) foram as doenças das veias, dos vasos linfáticos e dos gânglios linfáticos, não classificadas em outra parte (I80-I89), e as neoplasias malignas (C00-C97).

A incapacidade para o trabalho analisada de acordo com a potencial influência da idade mos- trou que a faixa etária de 40 a 49 anos apresentou maiores incidências de benefícios auxílio-doença, seguida daquela entre 30 a 39 anos (Tabela 3). O grupo de trabalhadores menor de 20 anos foi o que menos se afastou do trabalho no período, seguido daqueles com mais de 60 anos.

Praticamente todas as causas de auxíliosdoença na faixa etária menor de 20 anos foram do grupo das lesões, envenenamento e algumas outras consequências de causas externas (capítulo XIX). Entre 20 a 39 anos, nota-se que o grupo das lesões continua sendo o de maior incidência, contudo os grupos das doenças do sistema osteomuscular e do tecido conjuntivo (capítulo XIII) e dos transtornos mentais e comportamentais (capítulo V) também passam a apresentar relevância nessa faixa etária. A partir dos 40 anos, o grupo das doenças osteomusculares (capítulo XIII) se torna a mais importante causa dos benefícios.

Ao analisar a incidência de incapacidade para o trabalho segundo as UFs (Tabela 4), identificam-se os estados de Mato Grosso do Sul, Goiás, 
Tabela 2

Incidência de benefícios auxílio-doença concedidos aos trabalhadores do ramo Correios, segundo grupos da $10^{\mathrm{a}}$ revisão da Classificação Internacional de Doenças (CID-10), espécie de beneficio e sexo. Brasil, 2008.

\begin{tabular}{|c|c|c|c|c|c|c|c|c|c|c|c|}
\hline \multirow[t]{3}{*}{ Grupos da CID-10 } & \multicolumn{6}{|c|}{ Espécie de benefício } & \multirow{3}{*}{$\begin{array}{l}\text { Total } \\
\text { M }\end{array}$} & \multirow[t]{3}{*}{ Total F } & \multirow{3}{*}{$\begin{array}{c}\text { RI } \\
\text { B31/B91 }\end{array}$} & \multirow{3}{*}{$\begin{array}{c}\text { RI } \\
M / F\end{array}$} & \multirow[t]{3}{*}{ Total } \\
\hline & \multicolumn{3}{|c|}{ B31 } & \multicolumn{3}{|c|}{ B91 } & & & & & \\
\hline & M & $\mathbf{F}$ & Total & $\mathbf{M}$ & $F$ & Total & & & & & \\
\hline M40-M54 - Dorsopatias & 37,3 & 40,4 & 37,3 & 19,1 & 18,7 & 18,6 & 56,4 & 59,1 & 2,0 & 1,0 & 55,9 \\
\hline S80-S89 - Traumatismos do joelho e perna & 33,4 & 9,4 & 27,0 & 34,1 & 12,6 & 28,2 & 67,5 & 21,9 & 1,0 & 3,1 & 55,1 \\
\hline M60-M79 - Transtornos dos tecidos moles & 17,2 & 32,6 & 20,6 & 21,6 & 44,1 & 26,6 & 38,8 & 76,8 & 0,8 & 0,5 & 47,2 \\
\hline M00-M25 - Artropatias & 29,2 & 25,4 & 27,7 & 9,7 & 7,5 & 9,0 & 38,9 & 32,9 & 3,1 & 1,2 & 36,7 \\
\hline S60-S69 - Traumatismos do punho e mão & 22,7 & 4,8 & 17,9 & 19,8 & 4,8 & 15,8 & 42,5 & 9,6 & 1,1 & 4,4 & 33,7 \\
\hline S90-S99 - Traumatismos do tornozelo e pé & 18,0 & 10,4 & 15,8 & 17,9 & 16,9 & 17,3 & 35,9 & 27,3 & 0,9 & 1,3 & 33,1 \\
\hline F30-F39 - Transtornos do humor & 18,2 & 45,2 & 24,4 & 2,4 & 6,7 & 3,4 & 20,6 & 51,9 & 7,3 & 0,4 & 27,7 \\
\hline S40-S49 - Traumatismos do ombro e braço & 14,3 & 3,2 & 11,3 & 14,9 & 3,2 & 11,8 & 29,2 & 6,4 & 1,0 & 4,5 & 23,1 \\
\hline F40-F48 - Transtornos neuróticos e somatoformes & 10,9 & 19,0 & 12,7 & 9,8 & 12,0 & 10,2 & 20,7 & 31,0 & 1,2 & 0,7 & 22,8 \\
\hline O20-O29 - Transtornos maternos relacionados & - & 21,9 & 21,9 & - & 0,0 & 0,0 & - & 21,9 & - & - & 21,9 \\
\hline \multicolumn{12}{|l|}{ à gravidez } \\
\hline S50-S59 - Traumas do cotovelo e antebraço & 10,2 & 3,7 & 8,4 & 10,2 & 4,8 & 8,7 & 20,3 & 8,6 & 1,0 & 2,4 & 17,1 \\
\hline $\begin{array}{l}\text { F10-F19 - Transtornos mentais por uso de substância } \\
\text { psicoativa }\end{array}$ & 20,7 & 1,9 & 15,7 & 1,2 & 0,0 & 0,9 & 21,9 & 1,9 & 17,4 & 11,7 & 16,6 \\
\hline K40-K46 - Hérnias & 17,6 & 7,2 & 14,7 & 0,5 & 0,0 & 0,4 & 18,1 & 7,2 & 38,0 & 2,5 & 15,1 \\
\hline 180-189 - Doenças das veias, vasos, gânglios linfáticos & 13,0 & 17,7 & 13,9 & 0,1 & 0,0 & 0,1 & 13,1 & 17,7 & 215,0 & 0,7 & 14,0 \\
\hline C00-C97 - Neoplasias malignas & 8,3 & 9,9 & 8,5 & 0,1 & 0,0 & 0,1 & 8,4 & 9,9 & 132,0 & 0,8 & 8,6 \\
\hline D10-D36 - Neoplasias benignas & 1,8 & 25,7 & 7,6 & 0,0 & 0,0 & 0,0 & 1,8 & 25,7 & - & 0,1 & 7,6 \\
\hline T90-T98 - Traumas, intoxicações e causas externas & 5,9 & 5,6 & 5,7 & 2,1 & 0,8 & 1,7 & 8,0 & 6,4 & 3,3 & 1,2 & 7,4 \\
\hline S70-S79 - Traumatismos do quadril e coxa & 4,3 & 0,8 & 3,4 & 4,8 & 1,3 & 3,9 & 9,1 & 2,1 & 0,9 & 4,3 & 7,2 \\
\hline Total & 389,8 & 412,1 & 387,4 & 181,8 & 144,5 & 169,1 & 571,5 & 556,6 & 2,3 & 1,0 & 556,5 \\
\hline
\end{tabular}

B31: auxílio-doença previdenciário; B91: auxílio-doença acidentário F: feminino M: masculino; RI: razão de incidência.

Nota: os valores do agrupamento O20-O29 para o sexo masculino e a RP B31/B91 para D10-D36 são inexistentes.

Santa Catarina e Rio Grande do Sul com as maiores incidências, contrapondo-se aos estados do Ceará, Pernambuco e Rondônia. Em todos os estados, predominou o benefício previdenciário (B31), e a razão previdenciária (B31/B91) foi maior no Rio Grande do Norte, Maranhão e Sergipe. Já o benefício acidentário foi proporcionalmente mais incidente em Roraima, Amapá, Santa Catarina e Rio Grande do Sul.

As UFs, quando analisadas segundo a despesa previdenciária mediana dos benefícios auxílio-doença, mostraram que, para o conjunto dos trabalhadores do ramo de atividade Correios, esse custo foi de R $\$ 1.847,00$. Por outro lado, $25 \%$ dos benefícios apresentaram despesa previdenciária inferior a $\mathrm{R} \$ 1.035,00$, contrapondo-se com os $25 \%$ daqueles com as maiores despesas, acima de R\$3.265,00. As UFs com as maiores despesas medianas foram Alagoas ( $\mathrm{R} \$ 2.371,00)$, Bahia ( $\mathrm{R} \$$ $2.368,00)$ e Espírito Santo (R\$ 2.353,00). Quanto à duração mediana dos auxílios-doença, esta foi, no geral, de 59 dias, com variação de 46 a 76 dias entre as UFs (Tabela 4).

A despesa previdenciária apresentou um aumento diretamente proporcional à faixa etária, no sexo masculino, para ambas as espécies de benefício, até os 59 anos de idade, havendo um discreto declínio para a faixa etária acima dos 60 anos (Tabela 5). Para o sexo feminino, a relação foi semelhante, exceto na faixa etária menor de 20 anos, que apresentou a maior despesa previdenciária no período.

No que se refere à duração dos benefícios, observa-se que, no geral, $74,2 \%$ destes duraram menos de 90 dias. Ao analisar esse indicador segundo a espécie de beneficio, houve maior duração entre os auxílios-doença acidentários, dentre os quais $71 \%$ apresentaram duração inferior a 90 dias, se comparados com $87 \%$ entre os previdenciários. Quanto ao sexo, a duração dos benefícios foi maior entre os homens do que entre as mulheres. 
Incidência de benefícios auxílio-doença concedidos aos trabalhadores do ramo Correios, segundo capítulos da 10ạ revisão da Classificação Internacional de Doenças (CID-10) e a faixa etária. Brasil, 2008.

\begin{tabular}{|c|c|c|c|c|c|c|c|}
\hline \multirow[t]{2}{*}{ Capítulos da CID-10 } & \multicolumn{6}{|c|}{ Faixa etária (anos) } & \multirow[t]{2}{*}{ Total } \\
\hline & $<20$ & $20-29$ & $30-39$ & $40-49$ & $50-59$ & $\geq 60$ & \\
\hline I- Doenças infecciosas & 0,0 & 5,0 & 8,3 & 6,7 & 6,1 & 3,7 & 6,3 \\
\hline II - Neoplasias & 0,0 & 6,7 & 12,1 & 31,2 & 27,7 & 22,1 & 17,1 \\
\hline III - Doenças do sangue & 0,0 & 0,2 & 1,1 & 0,0 & 0,9 & 3,7 & 0,6 \\
\hline IV - Doenças endócrinas & 0,0 & 1,0 & 3,7 & 3,2 & 3,3 & 3,7 & 2,7 \\
\hline V - Transtornos mentais & 0,0 & 58,9 & 95,1 & 90,8 & 47,5 & 11,0 & 72,7 \\
\hline $\mathrm{VI}$ - Doenças do sistema nervoso & 0,0 & 3,4 & 10,3 & 11,7 & 15,5 & 7,4 & 9,1 \\
\hline VII - Doenças do olho e anexos & 0,0 & 5,7 & 5,7 & 9,1 & 14,6 & 11,0 & 7,6 \\
\hline VIII - Doenças do ouvido & 0,0 & 0,7 & 1,8 & 2,4 & 2,8 & 3,7 & 1,7 \\
\hline IX - Doencas do aparelho circulatório & 0,0 & 10,5 & 21,1 & 42,4 & 62,0 & 33,1 & 28,4 \\
\hline X-Doenças do aparelho respiratório & 0,0 & 4,1 & 5,9 & 7,7 & 6,6 & 0,0 & 5,6 \\
\hline $\mathrm{XI}$ - Doenças do aparelho digestivo & 3,6 & 22,3 & 31,0 & 45,8 & 43,2 & 22,1 & 32,6 \\
\hline XII - Doenças da pele & 0,0 & 4,5 & 4,6 & 3,7 & 4,7 & 3,7 & 4,2 \\
\hline XIII - Doenças osteomusculares & 3,6 & 75,1 & 161,2 & 215,8 & 165,5 & 40,5 & 143,7 \\
\hline XIV - Doenças do aparelho geniturinário & 0,0 & 9,6 & 17,8 & 17,6 & 16,0 & 11,0 & 14,5 \\
\hline XV - Gravidez, parto, puerpério & 0,0 & 13,9 & 15,2 & 2,1 & 0,0 & 0,0 & 8,7 \\
\hline XVII - Más-formações & 0,0 & 0,7 & 0,4 & 0,3 & 0,5 & 0,0 & 0,5 \\
\hline XIX - Causas externas & 134,6 & 254,6 & 224,0 & 180,6 & 104,8 & 25,8 & 195,8 \\
\hline$X X I$ - Exames de rotina & 0,0 & 3,1 & 5,7 & 3,2 & 7,1 & 0,0 & 4,3 \\
\hline Total & 141,9 & 480,3 & 624,9 & 675,4 & 529,3 & 202,5 & 556,5 \\
\hline
\end{tabular}

Nota: os valores do capítulo XV para o sexo masculino e a RP B31/B91 relativa ao capítulo XVII são inexistentes.

\section{Discussão}

Segundo a principal empregadora do ramo, no ano de 2008, mais da metade do efetivo da empresa era composto por carteiros. Estes, em conjunto com os atendentes e operadores de triagem e transbordo, são responsáveis pelas atividades operacionais da empresa e, naquele ano, correspondiam a cerca de $90 \%$ do efetivo 17 .

Com base nesses números, entende-se que a população em estudo é composta essencialmente por trabalhadores da área operacional, os quais exercem a atividade central da empresa. Quanto ao trabalho exercido pelo carteiro, em particular, trata-se de atividade que apresenta riscos substanciais de acidentes 15 , podendo justificar as elevadas incidências de auxílios-doença por lesões, envenenamento e algumas outras consequências de causas externas. Quase 50\% dessas lesões foram consideradas acidentes de trabalho, o que corrobora a presença de riscos inerentes a essa categoria de trabalhadores.

Apesar de novas tecnologias para aumentar a eficiência e melhorar a qualidade do serviço, a entrega de correio continua a ser um trabalho físico e intensivo, no qual o carteiro exerce, obri- gatoriamente, uma atividade repetitiva e solitária por longas horas, muitas vezes em condições sociais e ambientais desfavoráveis 20 . Tais situações - envolvendo pesos, tarefas físicas e trabalho monótono - aumentam o risco de problemas musculoesqueléticos, absenteísmo-doença e estão associadas a risco de quedas, fraturas e lesões ${ }^{15}$. Nota-se que, nessa população, as lesões (capítulo XIX) corresponderam a 35,2\% do total de auxílios-doença, percentual acima do total desses benefícios no Brasil (33\%) 2 .

A elevada incidência de traumas nos braços e pernas pode estar associada às condições de trabalho e ao excesso de peso que o carteiro precisa carregar. Esse trabalhador, responsável pela entrega de correspondências em uma área prédelimitada, começa a jornada de trabalho com a separação das correspondências por região, uma atividade repetitiva e manual. Em seguida, ele precisa administrar um grande número de entregas por dia, e nem sempre há meios de transporte disponíveis. Como consequência, precisa carregar excesso de peso por longas distâncias, propiciando traumatismos e artropatias. Além disso, em muitas localidades no Brasil, as ruas são mal pavimentadas, com ladeiras ou de difícil 
Tabela 4

Incidência de benefícios auxílio-doença concedidos aos trabalhadores do ramo Correio, segundo a Unidade da Federação (UF), a espécie de benefício, a duração (em dias) e despesa previdenciária (em Reais) dos benefícios. Brasil, 2008.

\begin{tabular}{|c|c|c|c|c|c|c|}
\hline UF & B31 & B91 & $\begin{array}{c}\text { RI } \\
\text { B31/B91 }\end{array}$ & Total & $\begin{array}{c}\text { Despesa (R\$) } \\
\text { Mediana (quartis) * }\end{array}$ & $\begin{array}{c}\text { Duração (dias) } \\
\text { Mediana (quartis) * }\end{array}$ \\
\hline Mato Grosso do Sul & 603,19 & 214,22 & 2,82 & 817,40 & $1.596(808 ; 2.617)$ & $46(27 ; 74)$ \\
\hline Goiás & 629,20 & 186,55 & 3,37 & 815,75 & $1.333(787 ; 2.366)$ & $76(47 ; 109)$ \\
\hline Santa Catarina & 466,93 & 302,57 & 1,54 & 769,49 & $1.705(1.034 ; 2.887)$ & $59(41 ; 82)$ \\
\hline Rio Grande do Sul & 466,73 & 286,52 & 1,63 & 753,25 & $1.832(1.032 ; 3.129)$ & $59(37 ; 91)$ \\
\hline Mato Grosso & 517,79 & 215,19 & 2,41 & 732,98 & $1.395(680 ; 2.952)$ & $46(29 ; 72)$ \\
\hline Amazonas & 536,28 & 195,58 & 2,74 & 731,86 & $1.762(1.106 ; 2.664)$ & $56(39 ; 77)$ \\
\hline Alagoas & 501,36 & 212,70 & 2,36 & 714,06 & $2.371(1.340 ; 4.266)$ & $74(48 ; 104)$ \\
\hline Roraima & 369,94 & 323,70 & 1,14 & 693,64 & $1.864(1.420 ; 3.458)$ & $72(34 ; 90)$ \\
\hline Paraná & 465,33 & 198,01 & 2,35 & 663,35 & $1.622(941 ; 2.894)$ & $59(33 ; 96)$ \\
\hline Paraíba & 417,01 & 208,50 & 2,00 & 625,51 & $1.796(1.072 ; 3.073)$ & $46(30 ; 74)$ \\
\hline Rio de Janeiro & 412,21 & 175,12 & 2,35 & 587,32 & $2.125(1.204 ; 3.564)$ & $62(42 ; 90)$ \\
\hline Distrito Federal & 400,82 & 157,41 & 2,55 & 558,23 & 2.073. (1.169; 3.886) & $54(35 ; 79)$ \\
\hline Maranhão & 432,98 & 94,90 & 4,56 & 527,88 & $2.013(1.262 ; 3.737)$ & $62(39 ; 97)$ \\
\hline Sergipe & 427,28 & 98,60 & 4,33 & 525,88 & $1.991(997 ; 3.262)$ & $47(37 ; 73)$ \\
\hline Espírito Santo & 355,24 & 166,28 & 2,14 & 521,53 & $2.353(1.202 ; 4.024)$ & $76(47 ; 109)$ \\
\hline Pará & 315,08 & 178,94 & 1,76 & 494,02 & $2.313(1.502 ; 3.925)$ & $74(50 ; 101)$ \\
\hline Rio Grande do Norte & 418,42 & 66,42 & 6,30 & 484,84 & $2.018(1.129 ; 3.464)$ & $69(37 ; 91)$ \\
\hline Minas Gerais & 370,26 & 105,40 & 3,51 & 475,66 & $1.535(789 ; 2.874)$ & $50(30 ; 77)$ \\
\hline São Paulo & 324,23 & 139,04 & 2,33 & 463,27 & $1.939(1.066 ; 3.395)$ & $63(38 ; 100)$ \\
\hline Piauí & 323,90 & 114,72 & 2,82 & 438,62 & $2.076(1.097 ; 3.019)$ & $60(37 ; 77)$ \\
\hline Bahia & 274,94 & 145,11 & 1,89 & 420,05 & $2.368(1.272 ; 4.448)$ & $72(43 ; 112)$ \\
\hline Amapá & 219,91 & 183,26 & 1,20 & 403,18 & $1.575 ;(955 ; 2.077)$ & $60(14 ; 78)$ \\
\hline Ceará & 271,69 & 123,89 & 2,19 & 395,58 & $1.519 ;(860 ; 2.301)$ & $58(34 ; 80)$ \\
\hline Pernambuco & 228,00 & 130,28 & 1,75 & 358,28 & $1.549(870 ; 3.020)$ & $57(36 ; 85)$ \\
\hline Rondônia & 199,42 & 94,46 & 2,11 & 293,89 & $1.107(751 ; 2.6730$ & $49(32 ; 85)$ \\
\hline Brasil & 387,39 & 169,13 & 2,29 & 556,51 & $1.847(1.035 ; 3.265)$ & $59(36 ; 91)$ \\
\hline
\end{tabular}

B31: auxílio-doença previdenciário; B91: auxílio-doença acidentário; RI: razão de incidência.

* Quartis 1 e 3.

Nota: os estados do Acre e Tocantins não foram listados. Eles apresentaram um e cinco benefícios auxílio-doença,

respectivamente.

acesso, expondo os carteiros a riscos de quedas e torções.

O risco de acidente por mordedura de cães também é elevado. Em estudo australiano, ataques feitos por cães durante a atividade profissional ocorreram em $55 \%$ da amostra de trabalhadores dos Correios em Brisbane (Austrália) 21.

Ademais, o maior risco de acidentes observado na atividade postal está relacionado não apenas à questão física, mecânica e ergonômica, mas também se associa com a disseminada violência social. O risco de assaltos é um exemplo desse problema nesse ramo de atividade 15 , que apresenta menos segurança e proteção quando comparada a outras instituições que lidam com objetos ou documentos de valor, como os ban- cos. Além disso, o papel dos correios mudou consideravelmente para oferecer uma gama diversificada de produtos e serviços, incluindo serviços bancários, transferência de dinheiro eletrônico, seguros, serviços de pensões, pagamento de contas, passaporte e pedidos de licenciamento 22,23. Assim, esse trabalhador precisa rotineiramente transportar objetos de valor, sendo um alvo em potencial para assaltos e furtos, agravando o estresse na profissão.

Diante disso, nota-se que o contexto da globalização repercutiu diretamente no trabalho dos carteiros, dando origem à insegurança no trabalho, insatisfação, conflito interno e aumento da carga laboral 15. É possível que essa nova realidade contribua para elevada incidência de 
Despesa previdenciária (em Reais) e duração (em dias) dos benefícios auxílio-doença concedidos aos trabalhadores do ramo Correios, segundo a idade, espécie do benefício e o sexo. Brasil, 2008.

\begin{tabular}{|c|c|c|c|c|}
\hline \multirow[t]{4}{*}{ Faixa etária (anos) } & \multicolumn{4}{|c|}{ Sexo masculino } \\
\hline & \multicolumn{2}{|c|}{ B31 } & \multicolumn{2}{|c|}{ B91 } \\
\hline & Duração (dias) & Despesa (R\$) & Duração (dias) & Despesa (R\$) \\
\hline & Mediana (quartis) * & Mediana (quartis) * & Mediana (quartis) * & Mediana (quartis) * \\
\hline$<20$ & $53(31,3 ; 102)$ & $861(580 ; 1.542)$ & $50(42 ; 106)$ & $1.012 ;(581 ; 1.466)$ \\
\hline $20-29$ & $59(36 ; 91)$ & $1.231(761 ; 2.033)$ & $68(45 ; 100)$ & $1.314(837 ; 1.972)$ \\
\hline $30-39$ & $60(34 ; 89)$ & $1.677(979 ; 2.768)$ & $63(41 ; 97)$ & $1.779(1.051 ; 2.890)$ \\
\hline $40-49$ & $59(37 ; 88)$ & $2.498(1.442 ; 4.155)$ & $67(45 ; 98)$ & $2.894(1.695 ; 4.502)$ \\
\hline $50-59$ & $63(37 ; 100)$ & $3.481(1.918 ; 5.738)$ & $61(43 ; 89)$ & $3.313(1.862 ; 5.117)$ \\
\hline$\geq 60$ & $66(45 ; 111)$ & $3.104(1.703 ; 6.488)$ & $78(41,5 ; 130)$ & $2.850(2.334 ; 5.346)$ \\
\hline \multirow[t]{4}{*}{ Faixa etária (anos) } & \multicolumn{4}{|c|}{ Sexo feminino } \\
\hline & \multicolumn{2}{|c|}{ B31 } & \multicolumn{2}{|c|}{ B91 } \\
\hline & Duração (dias) & Despesa (R\$) & Duração (dias) & Despesa (R\$) \\
\hline & Mediana (quartis) * & Mediana (quartis) * & Mediana (quartis) * & Mediana (quartis) * \\
\hline$<20$ & $86(19 ; 199)$ & $2.299(263 ; 2.753)$ & - & - \\
\hline $20-29$ & $44(27 ; 75)$ & $976(566 ; 1.746)$ & $59(36 ; 98)$ & $1.266(798 ; 2.166)$ \\
\hline $30-39$ & $48(30 ; 83)$ & $1.423(804 ; 2.728)$ & $60(40 ; 88)$ & $1.601(1.041 ; 2.422)$ \\
\hline $40-49$ & $47(30 ; 78)$ & $1.799(1.010 ; 3.256)$ & $68(46 ; 87)$ & $2.935(1.952 ; 4.697)$ \\
\hline $50-59$ & $51(30 ; 88)$ & $1.946(1.094 ; 3.558)$ & - & - \\
\hline$\geq 60$ & $66(21 ; 172)$ & $1.918(320 ; 3.543)$ & - & - \\
\hline
\end{tabular}

B31: auxílio-doença previdenciário; B91: auxílio-doença acidentário.

* Quartis 1 e 3

transtornos mentais e comportamentais e das doenças do sistema osteomuscular e do tecido conjuntivo, todos notadamente influenciados por fatores psicossociais, estresse na atividade funcional e formas de organização dos processos de trabalho 12,24,25,26,27,28,29,30.

Some-se a isso o fato de que o progresso tecnológico intenso interferiu na atividade da empresa e, objetivamente, no trabalho do carteiro. Esse ramo de atividade passou por várias mudanças internas e externas, com destaque para novas exigências dos clientes, ampliação dos serviços e produtos, estimulando importantes transformações organizacionais em nível regional, nacional e global, necessárias para a própria sobrevivência dos Correios no novo mercado.

Entre essas mudanças, está a inserção da presença feminina no setor postal, tradicionalmente um ambiente de trabalho dominado por homens. Segundo os resultados da presente pesquisa, as doenças do sistema osteomuscular e os transtornos mentais foram mais incidentes nas mulheres, independentemente da espécie de benefício. Outros estudos também demonstraram maior associação dessas causas de incapacidade para o trabalho com o sexo feminino 12,30,31. Em adição, globalmente, a maior participação feminina na força de trabalho postal pode ser vista como uma ameaça ao status quo, possivelmente dando origem a assédio sexual, vitimizações por razões de gênero e comportamento machista e grosseiro para com colegas do sexo feminino ${ }^{15}$, sugerindo que esse ambiente possa ter repercussão na saúde dos trabalhadores, em particular, das trabalhadoras.

No geral, as mulheres apresentaram maiores incidências de benefícios previdenciários (B31), e os homens, de acidentários (B91). É possível que as patologias com maiores incidências entre as mulheres (doenças do sistema osteomuscular e os transtornos mentais) tenham menor relação com a atividade laboral, quando comparadas com as patologias mais incidentes no sexo masculino, tais como as lesões 32 . Enquanto as lesões são agravos agudos, facilmente associadas com um momento e local, aqueles dois grupos de doenças costumam ser agravos crônicos, o que dificulta o estabelecimento do nexo técnico entre o agravo e o trabalho ${ }^{32}$. Além disso, num ambiente machista, pode haver uma subcaracte- 
rização das patologias relacionadas ao trabalho que acometem as mulheres, ao se supor que, por preconceito de gênero, a paciente feminina seja "fraca”, não considerando a atividade laboral relevante no processo da doença.

Outra distinção entre os gêneros, já descrita na literatura 7,30 , foi a elevada razão de masculinidade para os transtornos mentais e comportamentais decorrentes do uso de substância psicoativa. Existe uma associação do uso de bebidas alcoólicas com a confraternização, virilidade e o trabalho, contexto social que poderia justificar essa diferença 7 .

A maior incidência de benefícios auxílio-doença na faixa etária de 40 a 49 anos é corroborada por diversos estudos, os quais demonstram uma correlação direta entre as taxas de benefícios e o envelhecimento 2,32. Em oposição, a baixa incidência desses benefícios em trabalhadores com menos de 20 e acima de 60 anos pode estar associada ao fato de esses grupos apresentarem mais afastamentos de curta duração ( $<15$ dias), não analisados nesse estudo. Esses afastamentos mais curtos podem estar relacionados com o pouco tempo de serviço no caso dos mais jovens; o tipo de atividade executada por aqueles acima de 60 anos, além de este segundo grupo ser constituído quase que exclusivamente pelo sexo masculino, o qual apresenta, na grande maioria das pesquisas, menor taxa de afastamento 2,30,31,33,34. Pesquisas já demonstraram que, entre jovens, há uma maior incidência de afastamentos de curta duração ${ }^{3}$ e que, talvez, trabalhadores mais velhos também optem por afastamentos mais curtos ou apresentem uma carga de trabalho diferenciada por estarem próximos a se aposentar; é possível, ainda, que, entre os mais velhos, fatores como a experiência sejam mais valorizados do que a capacidade física.

O perfil de afastamento entre os trabalhadores do ramo Correios foi distinto conforme as faixas etárias. Em jovens, as lesões foram praticamente a única causa de afastamento por auxíliosdoença, o que pode sugerir uma subnotificação de outros agravos de saúde nesse grupo. Porém, à medida que essa população envelhece, as doenças do sistema osteomuscular e os transtornos mentais surgem como importantes diagnósticos desses benefícios 33. Considerando a atividade do carteiro como possível fator contribuinte para essas patologias, a associação do envelhecimento e o maior tempo de serviço na empresa podem justificar esses resultados.

Quanto às diferenças entre os estados brasileiros, as grandes variações entre as incidências de incapacidade para o trabalho encontradas podem estar associadas às diferenças culturais e administrativas regionais. Em localidades com menor número de trabalhadores, a repercussão do afastamento no trabalho é maior, e essa realidade poderia influenciar na decisão por um afastamento superior a 15 dias.

As maiores incidências de benefícios auxíliodoença identificadas nos estados das regiões Sul e Centro-oeste podem ser, em parte, explicadas por uma melhor assistência médica aos trabalhadores, possivelmente contribuindo para uma maior notificação dos casos existentes, bem como por maior organização sindical. Em contrapartida, houve baixa notificação de casos nos estados do Ceará, Pernambuco e Rondônia, além de menor vinculação entre benefícios e trabalho no Rio Grande do Norte, Maranhão e Sergipe. Esses últimos resultados podem estar associados ao precário apoio à saúde do trabalhador, assim como à baixa organização de classe.

Acre e Tocantins não foram estudados individualmente, pois apresentaram, respectivamente, de um a cinco auxílios-doença durante o ano de 2008. Esse resultado provavelmente deveu-se ao baixo número de trabalhadores existentes nesses estados.

Comparando-se com a despesa média relacionada a auxílios-doença por acidente de trabalho no Brasil, em 2008 (R $2.181,00){ }^{32}$, a despesa média com esses benefícios para os trabalhadores do ramo de atividade Correios foi $15,3 \%$ mais baixa que a média nacional.

O aumento da despesa previdenciária foi proporcional ao envelhecimento da população de trabalhadores, o que pode decorrer da maior duração dos auxílios-doença e do maior saláriobenefício médio na faixa etária de trabalhadores mais velhos 32 . A maior despesa previdenciária evidenciada entre menores de 20 anos do sexo feminino também foi associada à elevada duração dos benefícios nessa faixa etária.

Os auxílios-doença acidentários tiveram maior duração quando comparados com os previdenciários. Considerando que os afastamentos de mais longa duração costumam ser diretamente associados a quadros clínicos de maior severidade 35 , é possível inferir que a atividade ocupacional proporcionou quadros clínicos mais graves.

Este estudo permitiu a análise do perfil do absenteísmo-doença dos carteiros no Brasil, em 2008, e reforça a importância desse tema na atualidade. Certamente, o meio mais eficaz para evitar o processo de adoecimento ocupacional é a prevenção. Os resultados encontrados nesta pesquisa demonstram que é necessária a aplicação de medidas preventivas mais eficazes nesse ramo de atividade, em particular nas atividades operacionais, com investimento em melhores condições de trabalho no setor postal. 


\section{Resumen}

Se analizó el perfil de los trabajadores de Correos en Brasil que recibieron subsidios por enfermedad en 2008. Los datos proceden de las bases de datos del Sistema Único de Beneficios y del Registro Nacional de Información Social. La incidencia fue de 556,5 subsidios de enfermedad por cada 10.000 empleados y las principales causas de solicitud fueron las lesiones, las enfermedades musculoesqueléticas y los trastornos mentales. Las partes del cuerpo, cuyas lesiones fueron más comunes, eran: la rodilla y la pierna, la muñeca y la mano, el pie y el tobillo, el hombro y el brazo, con mayor incidencia en los hombres. Las mujeres fueron las más afectadas por las enfermedades osteomusculares y los trastornos mentales. La duración del subsidio de enfermedad fue mayor en los hombres y hubo un aumento de la incidencia de este subsidio con el envejecimiento. El gasto en pensiones fue de $R \$ 1.847,00$ y los estados de Mato Grosso do Sul, Goiás y Santa Catarina fueron los de más alta incidencia. Es posible que la actividad postal presente riesgos adicionales de accidentes en las extremidades.

Servicios Postales; Absentismo; Enfermedades

Profesionales; Salud Laboral

\section{Colaboradores}

F. A. N. Mascarenhas participou do projeto, análise de dados, discussão, interpretação dos resultados, redação e análise final do artigo. A. Barbosa-Branco participou do projeto, análise de dados, discussão, interpretação de resultados e revisão final do artigo.

\section{Agradecimentos}

Ao Ministério da Previdência Social, em particular a Paulo Rogério Albuquerque-Oliveira, pela disponibilidade dos dados necessários à realização da pesquisa.

\section{Referências}

1. International Labour Organization. Estimating the economic costs of occupational injuries and illnesses in developing countries: essential information for decision-makers. Geneva: International Labour Organization; 2012.

2. Barbosa-Branco A, Souza WR, Steenstra IA. Incidence of work and non-work related disability claims in Brazil. Am J Ind Med 2011; 54:858-71.

3. Voss M. Work and health: epidemiological studies of sickness absence and mortality with special reference to work environment, factors outside work and unemployment [Masters Thesis]. Stockholm: Karolinska Institutet, National Institute for Working Life; 2002.
4. International Labour Organization. The prevention of occupational diseases: World Day for safety and health at work. Geneva: International Labour Organization; 2013.

5. Soeren M, Aruna B, Bergamo G, Newberry SJ. A review of methods to measure health related productivity loss. Am J Manag Care 2007; 13:211-7.

6. European Agency for Safety and Health at Work. New and emerging risks in occupational safety and health. http://www.osha.europa.eu/en/pub lications/outlook/new-and-emerging-risks-in-oc cupational-safety-and-health-annexes (acessado em 02/Dez/2013). 
7. Barbosa-Branco A, Mascarenhas FAN, Pena LGQ Alcoolismo como fator de incapacidade para o trabalho: prevalência de benefício auxílio doença no Brasil, 2007. Com Ciências Saúde 2009; 20:123-34.

8. Brasil. Lei no 8.213 , de 24 de julho de 1991. Dispõe sobre os Planos de Benefícios da Previdência Social e dá outras providências. Diário Oficial da União 1991; 25 jul.

9. Oyeflaten I, Lie S, Ihlebæk C, Eriksen H. Prognostic factors for return to work, sickness benefits, and transitions between these states: a 4-year followup after work-related rehabilitation. J Occup Rehabil 2013; [Epub ahead of print].

10. Michie S, Williams S. Reducing work related psychological ill health and sickness absence: a systematic literature review. Occup Environ Med 2003; 60:3-9.

11. Ildefonso SA, Barbosa-Branco A, AlbuquerqueOliveira PR. Prevalence of temporary social security benefits due to respiratory diseases in Brazil. J Bras Pneumol 2009; 35:44-53.

12. Guo HR, Chang YC, Yeh WY, Chen CW, Guo YL. Prevalence of musculoskeletal disorder among workers in Taiwan: a nationwide study. J Occup Health 2004; 46:26-36.

13. Bergström G, Bodin L, Bertilsson H, Jensen IB. Risk factors for new episodes of sick leave due to neck or back pain in a working population: a prospective study with an 18-month and a three-year follow-up. Occup Environ Med 2007; 64:279-87.

14. Verbeek J, Ivanov I. Essential occupational safety and health interventions for low-and middle-income countries: an overview of the evidence. Saf Health Work 2013; 4:77-83.

15. Giga SI, Hoel H, Cooper CL. Violence and stress at work in the postal sector. Geneva: International Labour Organization; 2003.

16. Communication Workers' Union. Stress survey. London: Communication Workers' Union; 2001.

17. Empresa Brasileira de Correios e Telégrafos. Relatório empresarial 2008. http://www.correios.com. $\mathrm{br} /$ sobreCorreios/empresa/publicacoes/relato riosEmpresariais/default.cfm (acessado em 26/ Nov/2013).

18. Brasil. Decreto no 3.048, de 6 de maio de 1999 . Aprova o regulamento da Previdência Social, e dá outras providências. Diário Oficial da União 1999; 7 mai.

19. Conselho Nacional de Previdência Social. Anexo à Resolução CNPS no 1.101, de 16 de julho de 1998. Metodologia para avaliação e controle dos acidentes de trabalho. Diário Oficial da União 1998; 20 jul.

20. Commission for Labor Cooperation. Violence at work in North America. http://www.naalc.org/ english/pdf/violenceEN.pdf (acessado em 02/ Dez/2013).

21. Wilks J, Davey P, Andrews G. Dog attack injuries: an emerging issue in workplace health and safety. Journal of Occupational Health and Safety, Australia and New Zealand 1994; 10:359-62.
22. International Labour Organization. Conclusions on employment, employability and equal opportunities in the postal and telecommunications services. In: Note on the Proceedings: Tripartite Meeting on Employment, Employability and Equal Opportunities in the Postal and Telecommunications Services. Geneva: International Labour Organization; 2002. p. 27-30.

23. Bell S. The reform of India post: transforming a postal infrastructure to deliver modern information and financial services. Washington DC: World Bank; 2002.

24. Szeto ACH, Dobson KS. Mental disorders and their association with perceived work stress: an investigation of the 2010 Canadian Community Health Survey. J Occup Health Psychol 2013; 18:191-7.

25. Roelen CA, Koopmans PC, Hoedeman R, Bültmann U, Groothoff JW, van der Klink JJ. Trends in the incidence of sickness absence due to common mental disorders between 2001 and 2007 in the Netherlands. Eur J Public Health 2009; 19:625-30.

26. Cohidon C, Imbernon E, Gorldberg M. Prevalence of common mental disorders and their work consequences in France, according to occupational category. Am J Ind Med 2009; 52:141-52.

27. Torp S, Riise T, Moen BE. The impact of psychosocial work factors on musculoskeletal pain: a prospective study. J Occup Environ Med 2001; 43: 120-6.

28. Souza NSS, Santana VS. Incidência cumulativa anual de doenças musculoesqueléticas incapacitantes relacionadas ao trabalho em uma área urbana do Brasil. Cad Saúde Pública 2011; 27:2124-34

29. Darshan MS, Raman R, Sathyanarayana TSR, Ram D, Annigeri B. A study on professional stress, depression and alcohol use among Indian IT professionals. Indian J Psychiatry 2013; 55:63-9.

30. Barbosa-Branco A, Bültmann U, Steenstra I. Sickness benefit claims due to mental disorders in Brazil: associoations in a population-based study. Cad Saúde Pública 2012; 28:1854-66.

31. Bekker MHJ, Rutte CG, Van Rijswijk K. Sickness absence: a gender-focused review. Psychol Health Med 2009; 14:405-18.

32. Almeida PCA, Barbosa-Branco A. Acidentes de trabalho no Brasil: prevalência, duração e despesa previdenciária dos auxílios-doença. Rev Bras Saúde Ocup 2011; 36:195-207.

33. Vieira ER, Albuquerque-Oliveira PR, BarbosaBranco A. Work disability benefits due to musculoskeletal disorders among Brazilian private sector workers. BMJ Open 2011; 1:e000003.

34. Silva LS, Pinheiro TMM, Sakurai E. Perfil do absenteísmo em um banco estatal em Minas Gerais: análise no período de 1998 a 2003. Ciênc Saúde Coletiva 2008; 13:2049-58.

35. Barbosa-Branco A, Ildefonso SAG. Prevalência e duração dos benefícios auxílio-doença decorrentes de asma no Brasil em 2008. J Bras Pneumol 2012; 38:1-9.

Recebido em 21/Nov/2012

Versão final reapresentada em 16/Dez/2013

Aprovado em 19/Dez/2013 